\title{
Kernos
}

Revue internationale et pluridisciplinaire de religion grecque antique

9 | 1996

Varia

\section{La kalliphanie du monde (l'épiphanie du beau) dans le Timée}

\section{Anna Kélessidou}

\section{(2) OpenEdition}

\section{Journals}

Édition électronique

URL : http://journals.openedition.org/kernos/1183

DOI : 10.4000/kernos. 1183

ISSN : 2034-7871

Éditeur

Centre international d'étude de la religion grecque antique

Édition imprimée

Date de publication : 1 janvier 1996

ISSN : 0776-3824

Référence électronique

Anna Kélessidou, «La kalliphanie du monde (l'épiphanie du beau) dans le Timée », Kernos [En ligne], 9 | 1996, mis en ligne le 21 avril 2011, consulté le 03 mai 2019. URL : http://journals.openedition.org/ kernos/1183 ; DOI : 10.4000/kernos.1183 


\section{La kalliphanie du monde (l'épiphanie du beau) dans le Timée}



Timée, $55 \mathrm{~d}$ et $92 \mathrm{c}$

\section{À qui s'adresse le mythe vraisemblable de la beauté du monde}

Avant d'entamer toute question sur la démiurgie du Timée, on doit avoir présent à l'esprit que, la vérité résidant pour Platon dans l'être intelligible, inengendré et éternel, là où l'optique du philosophe est humaine, changeante, liée au temps, comme c'est le cas de la moralité dans la religion des Lois - où le culte (717a-b) concerne les citoyens réels (732e) - et le cas de l'origine de l'univers et de l'humanité, dont il est question dans la mythologie scientifique du Timée, Platon a recours à des probabilités, à un conte sensé, vraisemblable $(29 \mathrm{c}-\mathrm{d})$.

Il a été dit que «mythologiser » revient à « anthropomorphiser » et « rationaliser " ${ }^{1}$. Tout conte, en tant que tel, tient du concret. Le conte de la beauté du monde dans le Timée permet à Platon de s'adresser non plus à l'aristocratie intellectuelle - à qui est destiné le logos sur le Bien Suprême et sur la cité idéale dans la République ni seulement aux familiers « des méthodes de la science » (Timée, 53), des précisions mathématiques concernant l'âme du monde ou l'astronomie, mais à tous ceux qui, reconnaissant les limites de la nature humaine, utilisent «dans l'étude de la phusis» un raisonnement vraisemblable $(57 \mathrm{~d})$, s'en contentent et ne vont pas chercher " plus loin » (29d); à ceux qui, par manière de relâche, laissant de côté les discours relatifs aux réalités qui toujours sont et, considérant le vraisemblable qui s'attache au devenir, goûtent « un plaisir sans remords » pouvant mettre dans leur vie « un moment de récréation modérée et raisonnable » (59d) ${ }^{2}$. Cette paidia s'adosse à la paideia. Le mythe de la beauté du monde est adressé à tous ceux qui peuvent saisir le beau par les yeux porteurs de lumière (45b); comme dit le Pbèdre (250c-d) : la beauté est une réalité sur laquelle on a prise au moyen du " plus clair de nos sens », "elle-même brillant d'une supérieure clarté », seule

\footnotetext{
1 M.P. NILSSON, Geschicbte der griecbiscben Rellgion, Munich, 1941, I, p. 349, cité par H. JOLY, Le Renversement platonicien. Logos, Éptstémé, Polis, Paris, Vrin, 1980, p. 222, n. 78.

2 Tr. L. BRISSON, Platon, Timée/Critias, Paris, Flammarion, 1992, p. 165.
} 
ayant obtenu le « lot de pouvoir être ce qui est le plus en évidence et ce dont le charme est le plus aimable $»^{3}$.

\section{Le précepte de l'unité}

Vers 500 avant notre ère, Alcméon de Crotone affirmait : «ce qui fait que les hommes meurent c'est qu'il ne leur est pas possible de joindre le commencement et la fin » (D.K. V.S. B 2). Si l'on applique ce précepte de l'unité à la compréhension du Timée, en prêtant l'oreille au dire de Platon, on peut saisir l'essentiel de la démiurgie platonicienne, au lieu de le perdre à travers les méandres des problèmes interprétatifs, posés pour la plupart par notre rationalisme intransigeant ou notre souci d'identifications ${ }^{4}$.

Au début du Timée (19a-b), Platon, ayant résumé par la bouche de Socrate un entretien « de la veille » sur la représentation de la cité idéale, déclare son désir de passer de la description abstraite à l'acte; de voir les «beaux êtres vivants » non plus «figurés en peinture » ou - malgré le fait qu'ils sont réellement en $v^{5} \mathrm{e}^{5}$ - « se tenant en repos », mais mis en mouvement; aussi, après avoir entrepris de décrire, par le biais d'un mythe vraisemblable (30b), la naissance de notre monde «qui est un être vivant, pourvu d'une âme et d'un intellect » (ibid.), "vivant unique visible » (d), modèle concret et agissant du beau, termine-t-il son exposé sur l'univers par un éloge poétique de «ce


beaux, mortels et immortels.

Et l'on sait qu'au début du Timée (19c-e) Platon précise qu'il ne méprise pas la race des poètes, mais la mauvaise imitation, celle due à la difficulté « de bien imiter en actions un monde auquel on est étranger par l'éducation ». Au poète et pédagogue Platon «l'apparente fantaisie » de la « démiurgie » du monde du Timée permet de mettre «en relief l'unité des choses ${ }^{6}$ et de donner in concreto un paradigme à imiter, un modèle visible de vie d'ordre et d'harmonie. «Vouloir briser l'unité de ce qui est harmonieusement uni et beau c'est le fait d'un méchant», déclare le philosophe (41b), par la bouche de son Ouvrier divin qu'il dote de beauté et de bonté. La méchanceté a aussi trait à l'homme qui ne serait pas à même d'apprécier la beauté cosmique; en

3 Tr. L. ROBIN, Platon, Pbèdre, Paris, Les Belles Lettres; cf. Timée, 47a sq.

4

Voir par ex. (à propos du démiurge): R.J. WOOD, The demiurge and bis model, in CJ, 63 (1968), p. 255-258; D. KEYT, The mad craftsman on the Timaeus, in Pbilosophical Review, 80 (1971), p. 230-235; J.C. NILlES, Approcbe mythique du Bien, du pythourgos et du démiurge, in Revue internationale de Pbilosopbte, 40 (1986), p. 115-139, surtout p. 132 sq.; cf. L. BRISSON, Le Même et l'Autre dans la structure ontologlque du Tlmée de Platon, Paris, 1974; A. KÉLESSIDOU, La conception platonicienne du divin dans les Lois et le Timée et son rapport avec le Blen de la République, in Annuaire de la Faculté des Lettres de l'Université d'Athènes, 1971, p. 303-313.

5

Pour notre traduction, cf. Timée, $77 \mathrm{~b}$.

6 A. RIVAUD, Introduction au Timée, Platon, Oeuvres Complètes, X, Paris, Les Belles Lettres, 1963, p. 8. 
témoigne l'exégète de Platon, Plotin, qui, bien que soutenant l'idée d'un univers n'ayant eu d'autre «topos » que l'âme (Enn. IV, 3, 15, et II, 9, 7), se réfère ainsi à son maître : «Platon le dit... il n'est pas permis que périssent les êtres venus de Dieu » (Enn. II, 1, 5). La leçon platonicienne que Plotin retiendra surtout est que le monde sensible est un phénomène esthétique; que le mépris du monde est le propre du méchant ${ }^{7}$.

\section{La visée de la démiurgie platonicienne et le pouvoir du démiurge}

Ce qui précède n'insinue point que le monde, dans la mythologie du Timée, est une théophanie, comme dans la tradition néoplatonicienne, où « tout ce qui mérite à quelque degré le titre de réalité, n'est qu'un moment défini de l'effusion illuminatrice du Bien ${ }^{8}$. Dans la démiurgie platonicienne l'essentiel est la cosmophanie, la kalliphanie du monde et sa justification rationnelle; celle-ci porte sur trois rapports :

a) Un rapport de copie à modèle, où Platon relie ensemble « mimétisme, artificialisme et vitalisme », comme son « vocabulaire en témoigne $»^{9}:$ le divin démiurge, contemplant et utilisant un modèle de toutes les réalités, de perfection éternelle, le vivant en soi (30c-d), en reproduit forme et puissance, travaillant sur des réalités déjà données qu'il combine selon des lois d'ordre; tout ce qu'il réalise en procédant ainsi « est nécessairement beau » (Timée, 28a-b). «Si ce monde est beau», dit Platon, « il est clair (que son ouvrier) fixe son regard sur le modèle éternel...». Ce monde, «... la plus belle des choses qui sont nées » (29a), est fait «à la ressemblance du plus beau des êtres intelligibles et d'un Etre parfait en tout » (30d). De cette considération finaliste, on admet que le monde est un, son unité se déduisant de l'unité nécessaire de son paradigme (31a).

b) Le second rapport concerne la nature de l'artisan du beau et de son oeuvre; dans cette optique, la causalité du devenir et du monde réside dans la nature bienfaisante et réfléchissante du divin Ouvrier, dans sa bonté (29a) et sa providence (e) - dieu qui, en tant que tel, «ne nourrit d'envie à l'égard de qui que se soit » (29e) -, et dans la conviction, comme le dit Platon à propos de la forme sphérique de l'univers, qu'il y a mille fois plus de beauté dans le semblable que dans le dissemblable (33b).

Et l'on sait que Plotin, gêné par l'explication téléologique de la démiurgie du monde - la spontanéité caractérisant pour lui la causalité des hypostases considère que la production délibérée du cosmos, comme celle du fabricant des statues (Enn. II, 9, 4), n'est qu'une conception anthropomorphique de la divinité; que dans ce qui est éternel il n'y a point de place pour la délibération et le raisonnement (Enn. VI, 7, 3); enfin, que cette providence divine n'est pas

\footnotetext{
7 Voir Enn. II, 9, 16, Contre les Gnostiques.

8 E. GILSON, La Pbilosophie au Moyen Age, Petite Bibliothèque Payot, p. 82.

9 JOLY, op. cit. (n. 1), p. 228.
} 
« entièrement libre, Dieu dépendant nécessairement du monde, puisqu'il ne peut s'empêcher de le produire " (Enn. VI, 7,1$)$. Si nous nous sommes permis de nous arrêter un instant aux objections plotiniennes, c'est pour illustrer que Platon, préoccupé de faire apparaître la vérité de ses propos, sait, mieux que tout autre, user de toutes les ressources; qu'il se sert même de l'anthropomorphisme pour imposer et justifier sa thèse de la beauté de notre monde. Parlant de l'agir de son démiurge, le philosophe dit dans un langage juridique : " et onques ne fut permis, onques n'est permis au meilleur de rien faire, sinon le plus beau » (Timée, 30a-b) ${ }^{10}$. Aussi, précise-t-il, l'Ouvrier divin, après avoir réfléchi, "a mis l'intellect dans l'âme et l'âme dans le corps, pour construire l'univers, de façon à réaliser une œuvre qui fût, par nature, la plus belle et la meilleure possible ${ }^{11}$. "Ainsi donc», ajoute-t-il, " conformément à une explication qui n'est que vraisemblable, il faut dire que notre monde... est né tel par l'action de la providence du dieu » (ibid. b-c).

c) Le troisième rapport concerne l'artisan " de la suprême beauté » et la nécessité. La visée du Démiurge étant la constitution, par l'ordre (53b), du


la nécessité « d'orienter vers le meilleur la plupart des choses qui deviennent » (48a); qu'il superpose en face de la cause mécanique la causalité finaliste de la pensée (ibid). Les quatre éléments ayant reçu leurs figures du Démiurge, «par l'action des Idées et des Nombres », le Dieu, dit Platon, «a fait un ensemble, le plus beau et le meilleur» (53b). "Toutes les choses... nées par l'action de la nécessité, l'artisan de la suprême beauté et du meilleur les a prises pour la production de ce qui naît, au moment où il engendrait le dieu qui se suffit et qui est le plus parfait» $(68 \mathrm{~d}-\mathrm{e})^{12}$.

C'est à l'appui de ce dernier texte sur la divinité du monde engendré par le Démiurge et de certaines autres expressions platoniciennes que nous croyons pouvoir déchiffrer le passage $55 \mathrm{~d}$, qui concerne l'eikìs $\lambda$ ó $\gamma_{0} \varsigma$ révélateur pour Platon de la divinité et de l'unicité de notre monde. Platon, par la bouche de Timée, répond ainsi à la question de savoir s'il existe effectivement un monde unique, ou bien cinq : «L'indication que nous donnons ( $\tau$ ò $\mu$ غ̀v oủv $\delta \grave{\eta}$







Ce texte difficile à traduire, nous le lisons à peu près comme Luc Brisson; nous lisons $\theta \varepsilon$ có $^{13}$ et non $\theta \varepsilon o ́ s$, avec une virgule après $\mu \eta v v ́ \varepsilon 1 ;$ nous répétons ce verbe, comme sous-entendu, ainsi que l'infinitif eiv $\alpha$ l; nous comprenons donc $\theta \varepsilon o ́ v$ comme attribut du monde ( $\mu \eta v v ́ \varepsilon \imath$ eî́val $\theta \varepsilon o ́ v$ ), en rapprochant la

10 Tr. RIVAud, op. cit. (n. 6), p. 143.

Tr. L. BRISSON, Platon, Timée..., p. 119.

12 Tr. RIVAUD, modifiée, et empruntée, pour une part, à celle de BRISSON, op. cit. (n. 2), p. 181

13

F. PHILOP.; A.RIVAUD retient $\theta$ eós. 



$\mu \eta v v ́ o v \sigma ı$ (qui attestent que ces choses sont ainsi ou : qui attestent ces vérités),




révèle l'existence d'un monde-dieu, unique (55d, cf. 31a-b). Un autre discours, dit Platon, «prenant autre chose en considération pourrait juger qu'il en va autrement $»^{14}$.

Platon pense-t-il aux mondes en nombre indéterminé d'Anaximandre ou à la cosmologie de Xénophane $?^{15}$ « Mais laissons ce propos », dit le philosophe, qui clôt la question du nombre des univers pour passer à l'exposé des figures des éléments, montrant ainsi que ce qu'il vise ici est la révélation de l'unicité et de la divinité de notre monde; et ce n'est pas un dieu qui fait signe, ainsi que


que Platon invoque les dieux ${ }^{16}$, les prie pour que ses « propos soient... logiquement ordonnés » $(27 \mathrm{c}-\mathrm{d})$, ou une divinité protectrice pour qu'elle le sauve « des considérations absurdes » et le mène «à une conclusion cohérente »(48d), montre qu'il croit à la rationalité de son discours sur le monde. Rivaud $^{17}$ dit avec raison que l'eikós n'affaiblit pas l'autorité du récit platonicien, mais plutôt la renforce. C'est pourquoi nous avons traduit l'عikós du passage $55 \mathrm{~d}$ comme « raisonnable ». D'autre part, on n'a pas le droit de dire que dieu indique à un être humain l'unicité du monde, d'abord parce que dieu, ainsi que l'affirme Platon dans le Banquet, 203b, " ne se mêle pas à l'homme »; ensuite, parce qu'un tel comportement n'entre pas, comme le dit L. Brisson ${ }^{18}$, dans le Timée. Ce qui convient à dieu, Platon nous le dit en attribuant à son divin Ouvrier une action limitée et «ponctuelle », consistant dans la fabrication « dans l'univers» de ce qui «est susceptible de présenter un caractère éternel ${ }^{19}$; après quoi le Démiurge transmet ses pouvoirs aux dieux jeunes, ses aides, qu'ils ordonnent aussi de «gouverner le vivant mortel » avec le plus de beauté et de bonté qu'ils le pourraient» (42e). "Pour sa part», ajoute Platon à propos du Démiurge, « après avoir réglé tout cela, il resta dans son


être compris comme dans les passages de l'Apologie (27b) et de la République

\footnotetext{
14 Tr. BRISSON, op. cit. (n, 2), p. 158.

15 Voir par ex. Pseudo-PluTARQUE, Stomates, 2; DIOGÈNE LAËrCE, Vies, I, 19. Chez Xénophane pourtant il ne s'agit pas de la pluralité des mondes, mais de la répétition indéfinie des mêmes phénomènes, voir J.P. DUMONT, Les Présocratiques, Paris, Gallimard, 1988, p. 1221, n.3.

16 Cf. Philèbe, 25b, Lois, 887c.

17 op.cit. (n. 6), p. 11.

18 op. cit. (n. 2), p. 245, n. 423.

19 BRISSON, op. cit. (n. 2), p. 27.

20 RIVAUD traduit : «demeura dans son état accoutumé »; BRISSON : « resta dans le lieu où il séjournait habituellement ».
} 



«comme il convient ». L'expression pourrait être traduite: «selon sa coutume convenable », qui consiste à ne plus se mêler au mortel (se mouvoir) ${ }^{21}$. Ce qui convient à l'étbos d'un dieu dans le Timée est l'accomplissement, ou le gouvernement du monde avec «le plus de beauté possible».

La raison de cette kalliphanie du monde, la raison pour laquelle Platon revient dans le Timée de son dédain pour le monde sensible, réside dans la croyance fervente du philosophe qu'une « union étroite existe entre la nature humaine et la nature universelle ${ }^{22}$; dans sa conviction que «le microcosme qu'est l'homme doit prendre modèle sur le macrocosme ${ }^{23}$. Par l'âme intelligente, «semée par le démiurge qui a pris l'initiative de (la) faire venir à l'existence » - et non pas par ses aides (41c-d) -, et que dieu a fait cadeau à chacun de nous comme d'un génie divin, l'homme s'élève au-dessus de la terre, en raison de son affinité avec le ciel, car, "nous sommes une plante non point terrestre, mais céleste $\gg(90 \mathrm{a})$.

Platon - poète qui ne méprise pas les poètes (19d), mais les imitateurs de mauvais modèles, et qui loue les imitateurs du divin, fabricant d'un Démiurge-

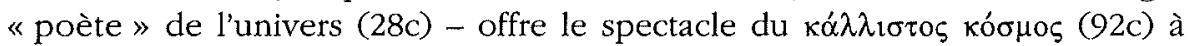
la contemplation de tous ${ }^{24}$, aux yeux humains «porteurs de lumière » $(45 \mathrm{~b})$, comme un modèle de permanence et de régularité à imiter $(90 \mathrm{c})$. La thèse qui considère la vue comme étant à l'origine de la philosophie ${ }^{25}$ - comme la thèse d'un modèle sensible qui pourrait déconcerter le pur idéaliste qui est trop peu poète ${ }^{26}$-, est conforme à l'esprit pédagogique de Platon; du penseur qui cherche partout des paradigmes de bon fonctionnement, fait feu de tout bois, relie esthétique, physique et éthique afin d'inciter les gens à devenir démiurges de leur propre perfection ${ }^{27}$.

Anna KÉLESSIDOU

Académie d'Athènes

Centre de Philosophie

14, Anagnostopoulou

GR - 10673 ATHÈNES

21 Cf. XÉNOPHANE B 26 : " (Au dieu) ne convient pas de se porter tantôt ici, tantôt ailleurs "; cf. W. JAEGER, À la naissance de la théologie, tr. de l'allemand, Les éd. du Cerf, 1966, p. 52 et 57.

22 RivaUd, op.cit. (n. 6), p. 10.

23 BRISSON, op. cit. (n. 2), p. 241, n. 254.

24 Cf. A.J. FESTUGIÈRE, Contemplation et vie contemplative selon Platon, Paris, Vrin, 1975.

25 BRISSON, op. cit. (n. 2), p. 246, n. 321

26 Cf. par ex. Hegel, voir J.-L. VIeIllard-BARON, Hegel, Leçons sur Platon, Paris, AubierMontaigne, 1976 , p. 110.

27 Cf. L. BRISSON, Les listes de vertus dans le Protagoras et dans la République, in Problèmes de la morale Antique, Université d'Amiens, Faculté des Lettres, Amiens, 1993, p. 91. 